\title{
Drug eluting stents in 2005
}

\section{A H Gershlick}

$l^{2}$ the last 3-5 years there has been a major shift in the management of symptomatic, obstructive coronary artery disease, with a move from predominantly surgical to increasingly a percutaneous approach. The perceived benefits of percutaneous coronary intervention (PCI) include patient convenience, ease of service expansion, and the fact that more complex lesions can now be undertaken through increases in operator skill and advances in balloon and stent technology. As a result there has been an exponential growth of PCI, with worldwide PCI rates exceeding surgical numbers for the last several years. The issue still to be decided is whether the percutaneous approach provides equivalent outcomes for all comers. This article will address the impact that drug eluting stents (DES) have had on improving the angioplasty result, what data are currently available to support different drug/platform combinations, and which issues still need to be resolved.

Improving the outcome after coronary stenting has been the goal since publication of the ARTS $^{1}$ and SOS $^{2}$ studies which, along with others, demonstrated the need for excess re-intervention in the stent arms in these studies of patients undergoing multivessel intervention. In the trials the differences were $14 \%$ and $15 \%$ at six months. Early studies with DES such as RAVEL ${ }^{3}$ suggested that such differences in re-intervention could be overcome, with very low restenosis rates being reported. However, as with all such developments it became clear that the patients treated in such early studies had lesions that could be regarded as relatively simple and therefore not representative of real world patients. The question to be asked in late 2004 early 2005 is: do the trials now represent real life and what are the current results?

\section{SIROLIMUS}

Sirolimus, also known as rapamycin, is a macrolide and is the metabolic substrate of the fungus Streptomyces hygroscopicus. It was discovered 20 years ago by Ayerst Research Laboratories in the soil on Easter Island (Rapa Nui) in the course of an antibiotic screening programme and for which, despite its effectiveness against yeast fungi, a licence was never applied for. However, it was found by chance to have an important role in immunosuppression and was thus developed for use in renal transplant rejection. Loaded on to stents at a dose of $180 \mu \mathrm{g}$ it was also found to have a pivotal role in inhibiting the processes that lead to cell proliferation after vessel wall balloon/stent injury. It does this by firstly binding to a receptor protein (FKBP12), with the rapamycin/FKB12 complex then binding to mTOR (mammalian target of rapamycin), preventing the interaction of mTOR with target proteins, such as regulatory tumour suppressor genes including P27, which are important in signalling pathways leading normally to cell proliferation. Such regulator genes control whether cells remain in the quiescent $G_{0}$ phase or move forward into the $G_{1}$ proliferative (and then $S$ ) phase. The early trial of rapamycin (RAVEL), while demonstrating efficacy, was criticised for the simple nature of the lesion tested, the so-called BENESTENT lesions, although others believed inclusion of such patients was entirely appropriate

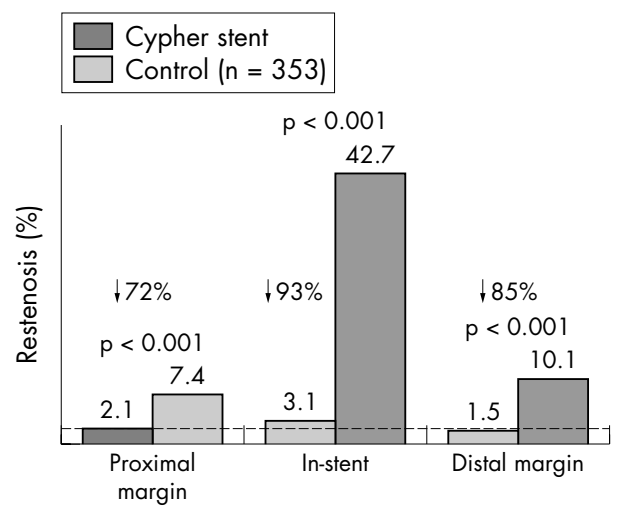

Figure 1 No edge effect in NEW-SIRIUS: quantitative coronary angiography peri-stent analysis. Pooled data from the E- and C-SIRIUS trials, 8 months angiographic and 9 months clinical follow up.

for initial demonstration in what was essentially a "proof of principle" trial. There were also concerns regarding apparent "excess effectiveness", with late loss ("difference in minimal lumen diameter from immediate post-stenting to follow up in $\mathrm{mm}^{\prime \prime}$ ) being so low (close to $0 \mathrm{~mm}$ ) as to suggest there was complete inhibition of tissue growth and therefore no stent coverage. The US based SIRIUS trial ${ }^{4}$ addressed more complex lesions and the late loss was subsequently greater resulting in an in-stent binary restenosis rate (BRR) of 3.2\%. However, there appeared to be a problem of tissue growth beyond either end of the stent (so-called "in-segment"defined as that occurring within $5 \mathrm{~mm}$ of each stent end), which reached $5.8 \%$ proximally. This phenomenon became known as the "edge effect" and was the result of balloon injury outside the stent with no drug to protect against the proliferative response. This, together with evidence of higher rates in those patients in the SIRIUS trial with small reference vessel diameters (target lesion revascularisation (TLR) rates $7.3 \%$ in those with mean reference vessel diameters $2.3 \mathrm{~mm}$ versus $1.8 \%$ in vessels with reference $3.3 \mathrm{~mm}$ ), and a higher rate of need for revascularisation in diabetics (in-stent BRR 8.3\%, in-segment BRR 17.5\%, and TLR $7.2 \%$ ) raised some concerns regarding robust efficacy in real life patients. The TLR rate of $13.9 \%$ in insulin dependant diabetics was particularly worrying, although the numbers in the post-hoc analysis were small.

The NEW-SIRIUS data addressed some of these issues. NEW-SIRIUS represents the pooled results of two trials: CSIRIUS $^{5}$ (the Canadian study of 100 patients), and E-SIRIUS ${ }^{6}$ (the European study of 352 patients). The trials were set up

Abbreviations: $B R R$, binary restenosis rate; $C A B G$, coronary artery bypass graft surgery; EPC, endothelial progenitor cell; ISR, in-stent restenosis; IVUS, intravascular ultrasound; LMS, left main stem; MACE, major adverse cardiac event; mTOR, mammalian target of rapamycin; $\mathrm{PCl}$, percutaneous coronary intervention; QCA, quantitative coronary angiography; TLR, target lesion revascularisation; TVR, target vessel revascularisation 
with identical entry criteria and end points, as well as having the same independent angiographic and monitoring committees. The primary end point was eight month angiographic outcome and there was a nine month clinical follow up. The results showed late loss of $0.18 \mathrm{~mm}$ in-stent and $0.17 \mathrm{~mm}$ outside the stent, indicating the so-called "edge effect" that was present in the SIRIUS trial had been overcome (presumably by a change in technique). It was thought that the edge effect in the SIRIUS trial may have been caused by the US operator practice of proximal dilatation after stent delivery, which had become routine with older non-conformable stents. In Europe conformable stents had arrived earlier and reduced the perceived need for post deployment dilatation. This was reinforced by there being the option of direct stenting in NEW-SIRIUS, which was performed in 30\% of patients in the trial (s). The proximal, in-stent, and distal ( $5 \mathrm{~mm}$ ) BRR for sirolimus loaded and control patients were, respectively, $2.1 \% \vee 7.4 \%, 3.1 \% \vee 42.7 \%$, and $1.5 \% \vee 10.1 \%$ (all $\mathrm{p}<0.001$ ), showing there was no "edge effect" (fig l). Overall the TLR for NEW-SIRIUS (the clinical end point) was $4 \%$ versus control $20.3 \%$. Clearly this study has become the new standard for sirolimus-eluting stents. In the small reference vessel subgroups results appear better than in SIRIUS, with ISR rates of $3.8 \%$ for vessels with mean size $2.2 \mathrm{~mm}$. TLR rates in diabetics is still around 7\%, however. On the other hand, overlapping stents were encouraged and the TLR rate in this group was only $2.5 \%$ versus $4.8 \%$ in the non-overlap patients.

There have also been concerns raised that polymer and/or drug could be removed during direct stenting, which accounts for about $30 \%$ of all percutaneous coronary interventional procedures, and therefore the DES efficacy could be affected. In NEW-SIRIUS this was found not to be the case with in-lesion restenosis in the pre-dilatation (sirolimus stenting group $7 \% \vee 2.4 \%$ in the patients undergoing direct sirolimus stent deployment). Finally, other recent data presented include longer term follow up of DES (sirolimus). The early RAVEL results appear to be maintained out to three years and the first-in-man $(n=45)$ patients appearing to have little loss in any of the measures of initial success out to four years, all of which does suggest that late restenosis catch-up is unlikely to be a problem.

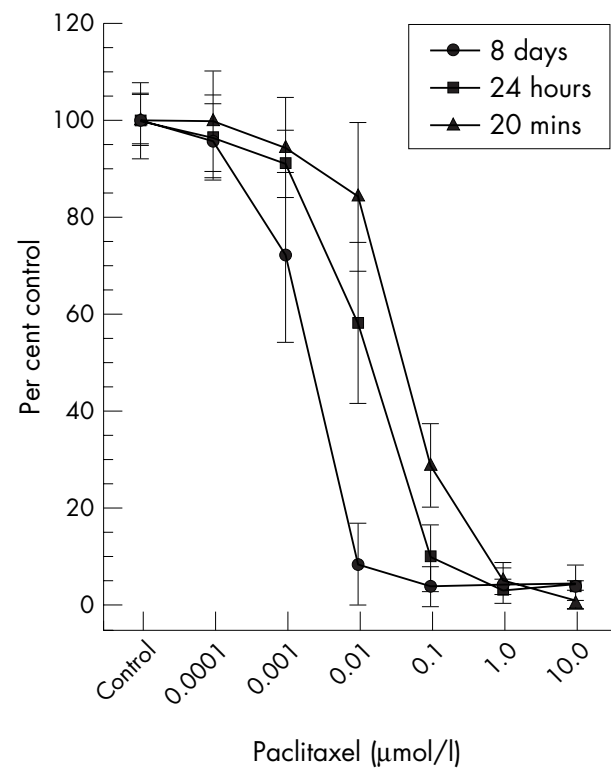

Figure 2 Inhibition of smooth muscle proliferation compared to control. Exposure time does not influence degree of inhibition. Reproduced with permission from Axel et al. ${ }^{7}$
Recently the issue regarding diabetics and DES has been addressed. At the 2004 TCT meeting, Sabaté presented the results of the "Diabetes" study. Angiographic restenosis in the sirolimus arm was 7.7\%, with TLR $7.5 \%$ and an overall major adverse cardiac event (MACE) rate of $11.3 \%$-all highly significantly improved $(\mathrm{p}=0.0001)$ over control bare metal stented patients.

\section{PACLITAXEL}

The potential toxic nature of the Yew tree was known to Julius Caesar, and the Pacific Yew Taxus brevifolia was used as a disinfectant, abortifacient, and cure for skin cancer by native Americans. Its contemporary therapeutic value was highlighted as part of a National Cancer Institute USA programme in which thousands of plants were screened for anticancer activity. In 1963 an extract from the bark of the Pacific yew (Taxus brevifolia), a relatively rare plant, was found to have cytotoxic activity against many tumours. It has specific properties against microtubules, promoting polymerisation of tubulin and inhibiting the disassembly of microtubules, which thus become very stable and dysfunctional, so inhibiting cell division. Cell replication is inhibited in the $\mathrm{G}_{0} / \mathrm{G}_{1}$ and $\mathrm{G}_{2} / \mathrm{M}$ phases. Other effects include those on cell motility, shape, and on transport between organelles. While it was recognised in the 1960s that the extract of the bark killed artificially preserved leukemia cells and was effective against advanced ovarian tumours, it was only in 1978 that it was shown that it killed cancer cells in a completely novel way by means of its microtubular stabilisation. It is these properties that make it a valuable agent for the treatment of restenosis when delivered locally on a stent.

The agent has been delivered in clinical trials in two ways, either with or without a polymer carrier. The importance of polymers for sustained delivery of agent versus the downside of long term polymer residency is an ongoing debate. For paclitaxel, which is very lipophylic and which was shown by $\mathrm{Axel}^{7}$ to be equally effective with either a 20 minute or eight hour exposure to the vessel wall, non-polymer delivery would appear a good option (fig 2). The ELUTES ${ }^{8}$ and ASPECT ${ }^{9}$ clinical trials tested similar dose ranges of paclitaxel applied directly to the stent without polymer. Both showed benefit in pre-trial determined measures of restenosis that reached levels of significance at dose density of approximately $3 \mu \mathrm{g} /$ $\mathrm{mm}^{2}$ of stent. These results led to investigators undertaking the pivotal US trial DELIVER 1, in which paclitaxel was loaded without polymer onto a different stent to that used in either of the other two previous trials. The results were disappointing with no significant benefit in the treated group. The reasons for the differences have still not been, and probably never will be, fully understood, but it has been suggested by O'Neill (PI DELIVER 1) that it may relate to non-uniform use of glycoprotein IIb/IIIa inhibitors across groups which influences thrombus formation and thus drug penetration, and presence of longer lesions. More likely the use of a different stent and/or the change in the drug/stent

Table 1 Differences and similarities of the patient profiles for TAXUS IV versus NEW-SIRIUS trials

\begin{tabular}{lll}
\hline & NEW-SIRIUS & TAXUS IV \\
\hline Reference vessel diameter & $2.61 \mathrm{~mm}$ & $2.79 \mathrm{~mm}$ \\
Lesion length & $14.8 \mathrm{~mm}$ & $13.4 \mathrm{~mm}$ \\
Diabetes & $20.0 \%$ & $23.4 \%$ \\
Stent/lesion length & $1.7 \mathrm{~mm}$ & $1.58 \mathrm{~mm}$ \\
Stent overlap & $34 \%$ & - \\
Angiography follow up & $87 \%$ & $43 \%$ \\
Direct stenting & $28 \%$ & - \\
\hline
\end{tabular}


production facility for loading led to an inability to accurately load the stent with the effective (ELUTES) dose. It would appear from the late loss results that not enough drug may have been loaded. No further development of a non-polymer stent is likely, which in an era of concerns about the longer term effect of residual polymer once the any drug has eluted, is disappointing. Recently a system for loading rapamycin directly onto the stent in the lab has been developed.

The difference in success between the non-polymer and the polymer coated paclitaxel (TAXUS) programme cannot have been more acute. The success of TAXUS $\mathrm{II}^{10}$ has been extended to more complex lesions in TAXUS IV. The differences and similarities of the patient profiles for TAXUS IV versus NEW-SIRIUS are shown in table 1 . The end point of TAXUS IV was clinical and thus the angiographic data can be misleading. The trial (end point) TLR rates were $3 \%$ versus $11 \%$ in controls $(p<0.0001)$ and the results appear robust even when there is an increase in lesion length or reduction in reference vessel diameter (fig 3 ). While the TLR rates for smaller vessels are similar in the sirolimus and paclitaxel trials to date $(3.4 \%$ for TAXUS IV $<2.5 \mathrm{~mm} v$ $3.6 \%$ for mean $2.5 \mathrm{~mm}$ in NEW-SIRIUS) there does seem to be better outcomes in the TAXUS diabetic patients, with the overall diabetic population having a TLR of $4.8 \%$ and the insulin dependant diabetics a TLR of $5.9 \%$. Further testament to the robust benefit of this drug/platform combination comes from the longer term preservation of outcome available from the TAXUS II study and data from TAXUS VI which tests longer lesions with multiple stenting. TAXUS VI results have been presented at PCR 2004 and demonstrate real robustness of biological effect in more complex lesions, although these data were with the moderate release polymer which is not the current commercially available formulation. Lesion length was $20.6 \mathrm{~mm}$, with a total stent length of $33.4 \mathrm{~mm}$, and American Heart Association/American College of Cardiology type C lesions in 55.6\%. A total of 446 patients were randomised: 219 into the TAXUS MR arm and 227 into the bare metal control arm of the study. TLR rate was $19.4 \%$ in the control group (44/227) and $9.1 \%$ in the TAXUS MR group (20/219) —representing a 53\% decrease in target vessel revascularisation (TVR), $\mathrm{p}=0.0027$ ). The difference was independent of classic risk factors for restenosis.

The concerns regarding the TAXUS programme lie with the revelation that up to $90 \%$ of drug is not released and remains bound within the polymer (hopefully completely and forever). Concerns regarding balloon deflation appear to have been overcome technically.

Is there any difference between the two currently commercially available stents? A comparator trial, the REALITY trial, has finished recruitment. A total of 1386

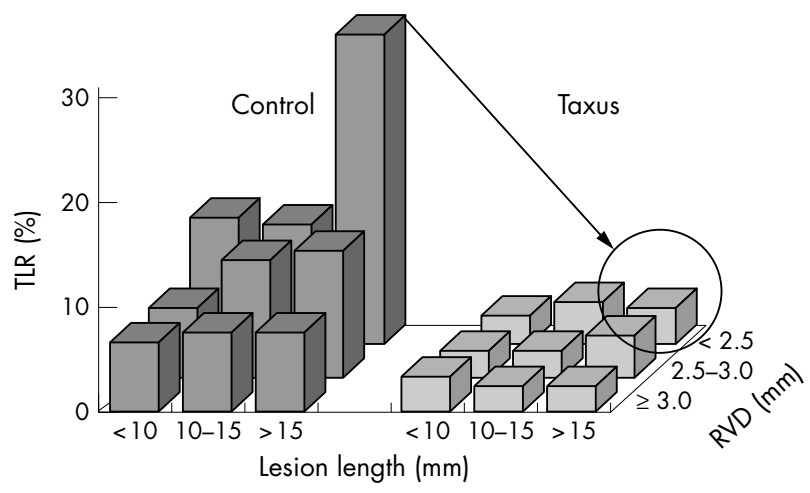

Figure 3 Good control of clinical effect of intimal hyperplasia with TAXUS stents irrespective of lesion length or reduction in vessel size. RVD, reference vessel diameter; TLR, target lesion revascularisation.

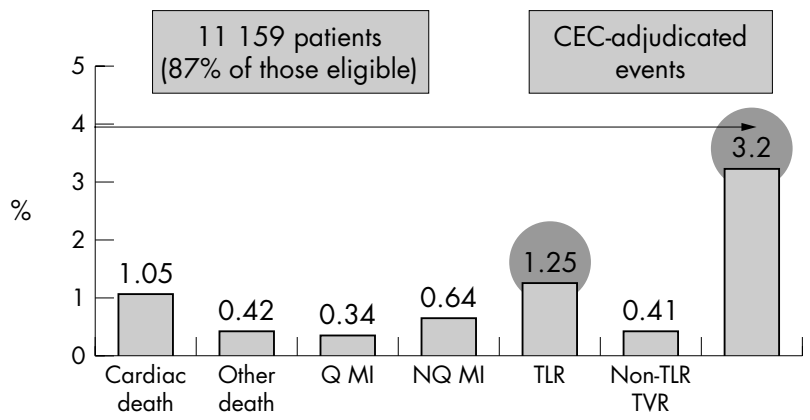

Figure 4 Real world studies: the e-Cypher study status December 2004. Six months follow up: major adverse cardiac events (MACE).

enrolled patients were randomised in a 1:1 fashion-Cypher versus TAXUS - with the primary end point of angiographic restenosis at eight months. This end point is important since previous individual stent clinical outcomes would suggest that if clinical data were to be the primary end point the differences would make the study underpowered. Interestingly stent deliverability, which was thought to be an issue with the Cypher stent, is reported in REALITY to be 98\% in both arms. Abstract results suggest equivalent TLR rates $(5.0 \%$ and $5.4 \%)$ although the late loss was less with Cypher $(0.09 \mathrm{~mm} v 0.31 \mathrm{~mm})$.

Until direct comparison trials are fully published there appears little to choose between currently available DES, although there appears, for what it is worth, to be a difference in mean percentage volume obstruction on intravascular ultrasound (IVUS) when all heterogenous studies are compared (RAVEL, SIRIUS, and E-SIRIUS mean $1.73 v$ TAXUS II slow release, TAXUS II fast release, TAXUS IV SR mean 9.26).

\section{REAL WORLD REGISTRIES}

For both of the currently available DES there is real world registry data, which although subject to selection bias criticism, is important information since many of the exclusions criteria of the randomised controlled trials included lesions treated in everyday practice (CTO, ISR, bifurcation disease, etc). The e-Cypher registry reached its recruitment target of 15000 patients (15 573) in August 2004. To date, 11159 (87\%) have been evaluated at six months. In these there are 1.3 stents per lesion and 30\% direct stenting. In total $48.7 \%$ of patients were so called treated off label (CTOs, saphenous vein graft (SVG), etc) Overall MACE was $3.2 \%$ with $6.4 \%$ SVG, $6.6 \%$ left main stem, non-diabetic $2.6 \%$, diabetic $4.6 \%$, and bifurcation $4.8 \%$ (fig 4 ). TLR rates are low (Fig 5) in all subsets compared to historical data. Furthermore clinically driven TVR in the Serruys RESEARCH registry of Cypher stent is 3.7\% $(\mathrm{n}=508)$.

In the WISDOM registry to date, 604 patients treated with TAXUS stents have MACE rates of $4.5 \%$ and TLR of $1.8 \%$, which appears to be better than the clinical trials. The MILESTONE II registry is designed to assess physician use and outcome by lesion subsets in 3000 patients. Data collection is ongoing, with 2000 patients enrolled in Europe.

\section{ONGOING DES PROGRAMMES}

New programmes in DES are important for a number of reasons. Firstly, as we extend the use of DES to encompass all lesions, more efficacious agents may be required. Stent delivered agents that are more lipophylic, have greater tissue penetration, or greater residency time may have true advantages. We may want agents that are more stable or have different release kinetics, or agents that work in completely different ways. Clinical trials of such stent 


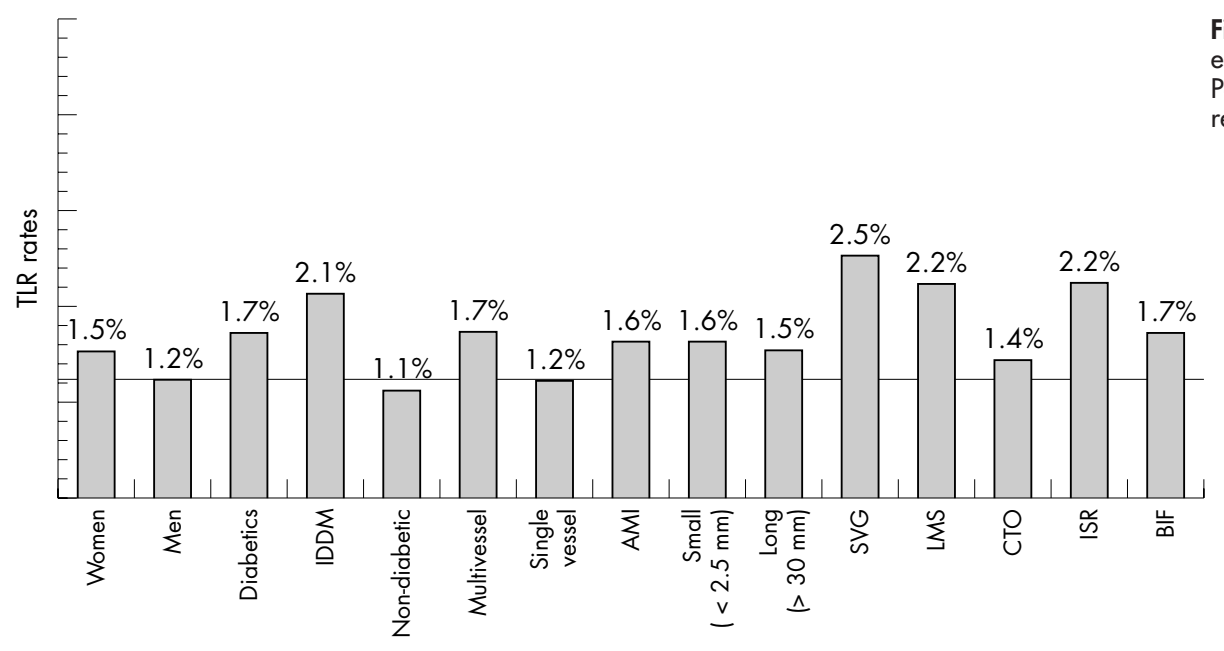

delivered new drugs are increasingly going to be required by the regulatory authorities to be tested against currently available DES, not bare metal stents.

Secondly we may wish for improved stent platforms. Treating complex lesions and making inroads into previous surgical cases will be dependant on technology and operator skills, not DES.

Thirdly, new DES mean competition which will lead to lower prices for this expensive technology.

\section{Sirolimus derivatives}

Sirolimus has three important chemical regions: the FK binding protein region, the non-protein binding region that influences physical properties region, and the mTOR binding domain (fig 6). C-43 sits in the first of these and it is substitution of the "HO" that has led to the second wave of agents. Thus the Medtronic programme consists of substitution at $\mathrm{C}-43$ with $5=\mathrm{N}$ ring formation. The now formed ABT578 $8^{11}$ has, in this programme, been adsorbed into
Figure 5 Real world studies: the e-Cypher study status December 2004 Patient subsets: 6 months target lesion revascularisation (TLR). a bio-neutral polymer (phosphorylcholine/Biocompatible) loaded to the Medtronic DRIVER stent. The trial series is named ENDEAVOR and is based on good laboratory and preclinical data suggesting ABT578 has potent effects on smooth muscle cell growth and inhibits intimal hyperplasia in a pig model at a dose of $10 \mu \mathrm{g} / \mathrm{mm}$ stent. The pilot ENDEAVOR I trial was a 100 patient study with one month MACE of one TLR and one non-Q wave infarct. Recently the one year outcomes have been reported and while there have been some concerns that the late loss is higher than expected (instent late lumen loss at 12 months of $0.58 \mathrm{~mm}$ ), and at a level that would normally be expected to lead to higher restenosis rates, the overall MACE rate remains at $2 \%$, with no further clinical events after four months. While this may relate to reference vessel size or the small size of the study, we need to wait for the pivotal ENDEAVOR II trial which finalised recruitment in the first quarter of 2004 and compared ABT578/Biocompatible (phosphorylcholine) polymer/Driver stent with bare metal stent in 1200 patients. The primary
Rapamycin chemical modifications

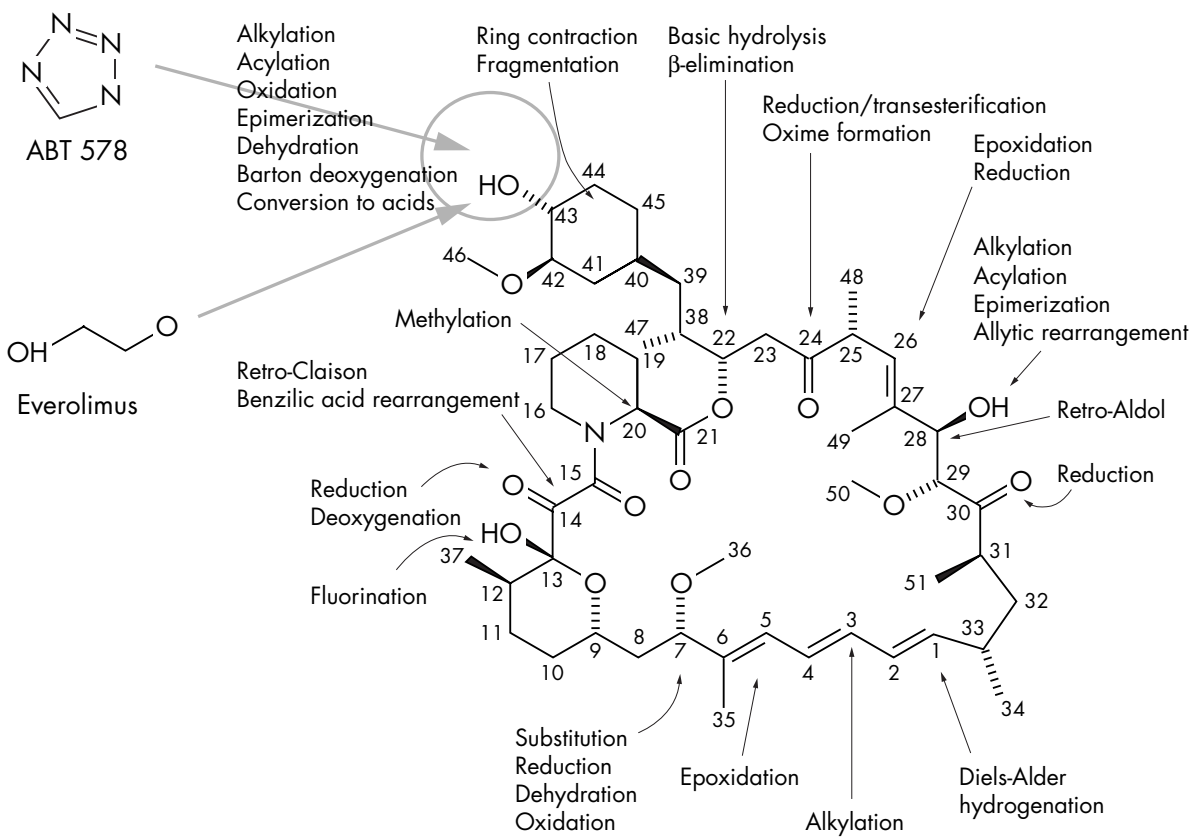

Figure 6 Rapamycin: substitution of C-43 results in sirolimus analogues. 


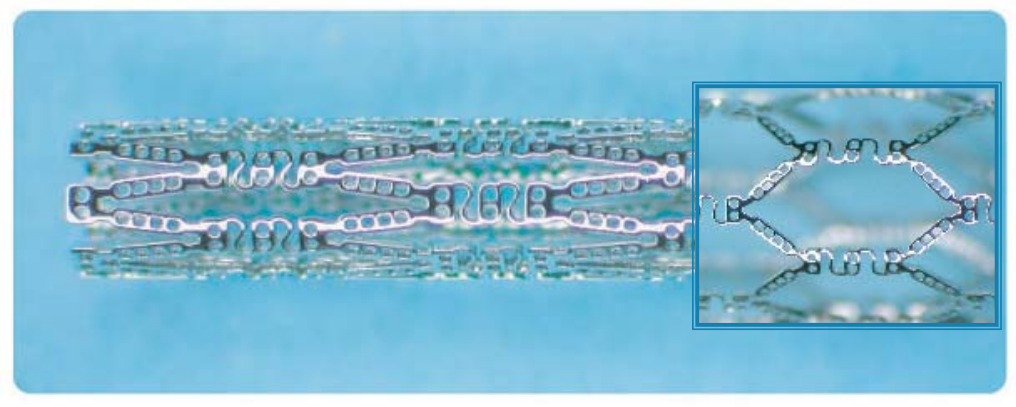

Controlled drug delivery from large, discrete, non-deforming reservoirs

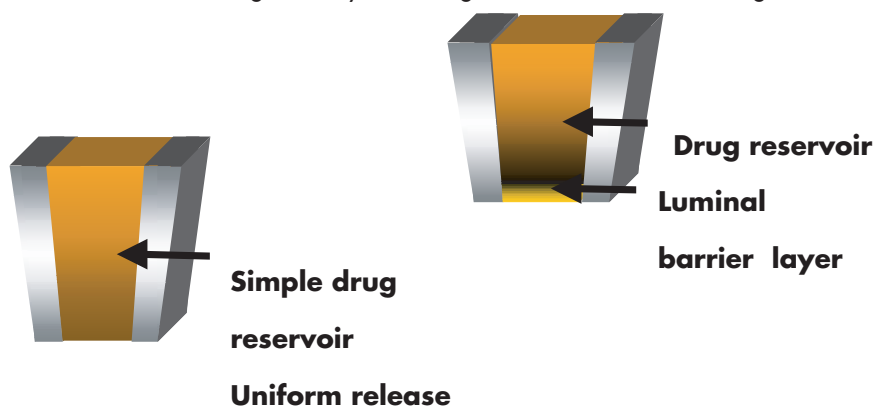

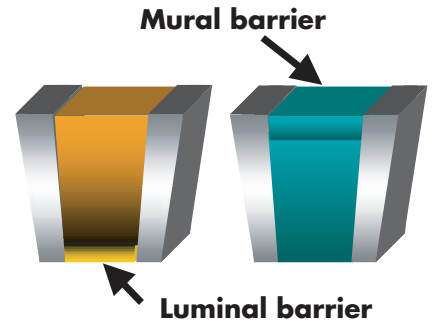

Two drugs, two wells

two directions

Figure 7 The Conor drug eluting stent design.

end point is target vessel failure (cardiac death, myocardial infarction, TVR) at nine months. There will be an angiographic follow up of the first 800 patients and IVUS in 300 in selected centres. Results were expected in fourth quarter of 2004. Early abstract results suggest excellent clinical outcome (TLR 4.6\% v 12.1\% controls) but interestingly with a "high" (compared to Cypher and Taxus) late loss of $0.62 \mathrm{~mm}$. Debate continues but deliverability may be important. ENDEAVOR III is planned as a 30 centre study across the USA of 436 patients randomised in a 3:1 fashion to ENDEAVOR stent or other drug eluting stent, probably Cypher. Again, because of likely small clinical differences, the primary end point will be angiographic "in-segment late loss" at eight months. ENDEAVOR IV is likely to be a comparison with the TAXUS stent and involve about 1000 patients. ENDEAVOR $\mathrm{V}$ is a registry.

Everolimus, like ABT578, is a sirolimus derivative with chemical substitution at C-43, but unlike ABT578 it is already available. Everolimus is being used in organ transplants and is reported to have better pharmacokinetics, tissue residency, and stability compared to its parent sirolimus compound. ${ }^{12}$ The Guidant Everolimus programme consists of the Biosensors Champion stent with a bio-absorbable polymer (polylactic acid PLA), which is already used in bioprostheses and has a high drug carrying potential. It breaks down to lactic acid. Safety and pilot efficacy data with this combination on the Champion stent has been published as the Future I and II studies ( $\mathrm{n}=42$ and $\mathrm{n}=64$ ), with MACE rates of $7.7 \%$ and $4.8 \%$ resp. and low "late loss" of $0.15 \mathrm{~mm}$. Future III will compare six month late loss in 800 patients randomised 3:1 to this drug/polymer/stent combination or to a bare metal Zeta stent, and FUTURE IV will compare this combination with a US Food and Drug Administration approved DES control $(\mathrm{n}=935$ randomised 2:1). The everolimus programme will also determine the value of this drug on a multi-link Vision stent with a durable stable polymer as carrier. However, recent information suggests that the company will indeed be going with the durable polymer/ Vision stent combination and promising results form the SPIRIT FIRST study have recently been presented. Total MACE at six months in the everolimus group was $7.7 \%(2 / 26$, one Q wave infarct and one TLR-PCI) compared to $21.4 \%$ MACE in the control arm (three TLR-coronary artery bypass graft surgery (CABG) and five TLR-PCI).

BiolimusA9 is yet another derivative of sirolimus and is claimed to be even more lipophylic, and from the Biosensors stent $>85 \%$ elutes into the tissue within eight hours. The drug delivered in a PLA polymer on the S-stent is being tested in a series of trials known as STEALTH. It is currently being tested on a conical ("bifurcation") stent, the DEVAXX.

Three other programmes are worth mentioning. The CONOR stent has a unique stent design (fig 7) that allows laser cut wells to be filled with polymer; with variation in barrier placement this stent can be configured to release drug to the vessel wall or towards the lumen, or both. Profile and stent deliverability appear good. The PISCES study is a pilot testing six different formulations with different doses released for different periods of time-for example, $10 \mu \mathrm{g}$ paclitaxel for between 10-30 days. Results reported recently suggest an overall 30 day MACE of $4.2 \%$, with the best formulations ( $10 \mu \mathrm{g} / 30$ day and $30 \mu \mathrm{g} / 30$ day) to be tested on a cobalt chromium Conor stent platform in the EuroSTAR trial.

The SCEPTER trial also compares two formulations of paclitaxel with 260 patients enrolled and quantitative coronary angiography (QCA) results are favourable. There will be an IVUS substudy.

Endothelial progenitor cell (EPC) capture has been proposed as a means of enhancing re-endothelialisation post-stent trauma, with all the advantages this brings of reduced restenosis (endothelial cells have potent smooth muscle cell inhibitory actions) and thrombosis. Michael Kutryk from Toronto is heading up a programme that involves covering stents with antibody to CD-34, which is a 
Endothelial progenitor cells

(EPCs)

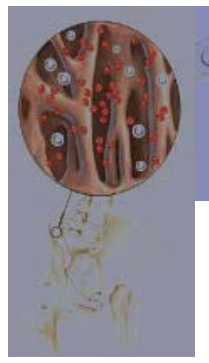

Circulating bone marrow derived endothelial progenitor cells

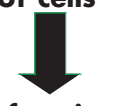

Mature, functional endothelial cells
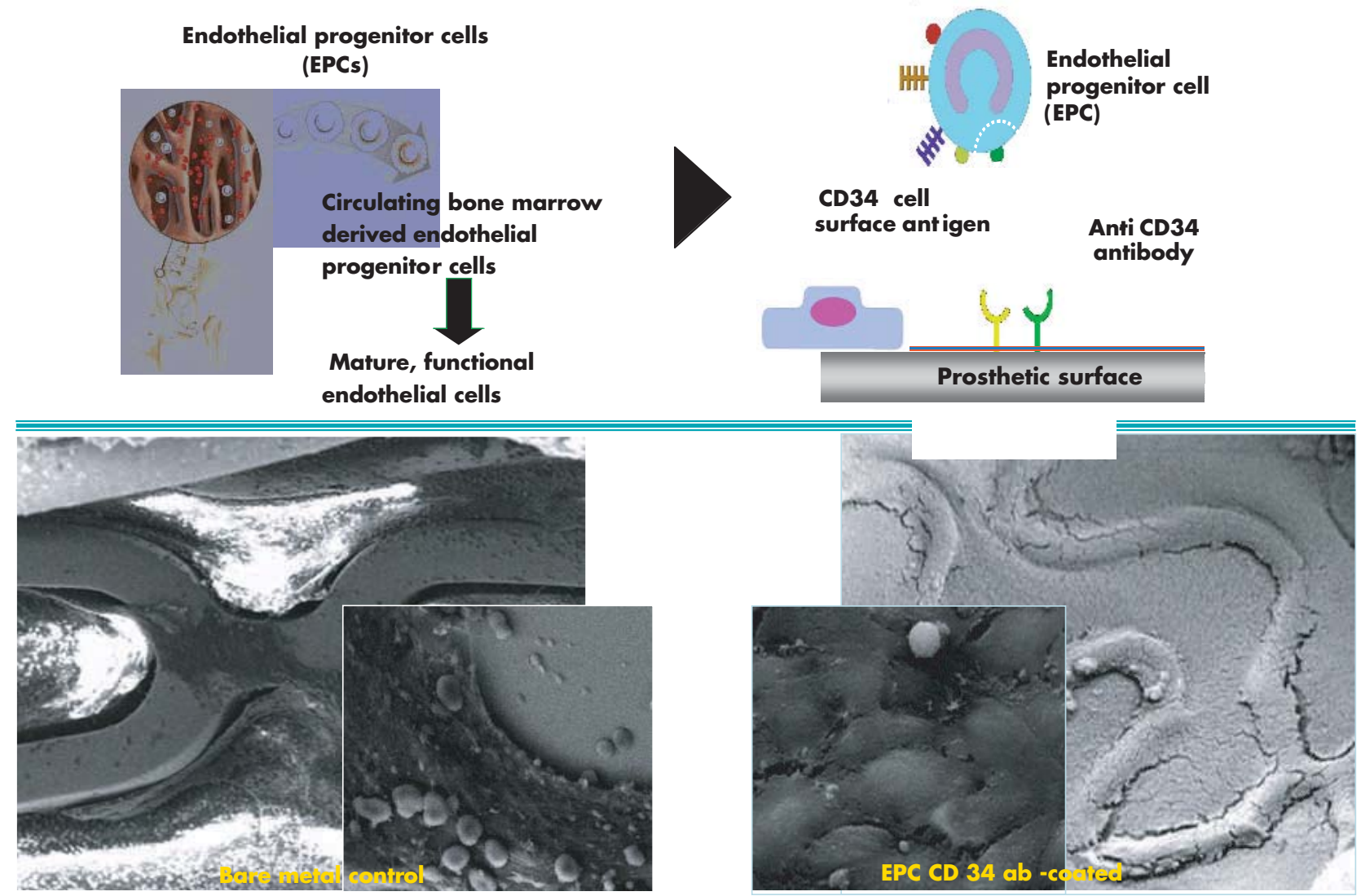

Figure 8 Endothelial progenitor cell technology.

\section{Pattern of restenosis \\ in SIRIUS $(n=31)$}

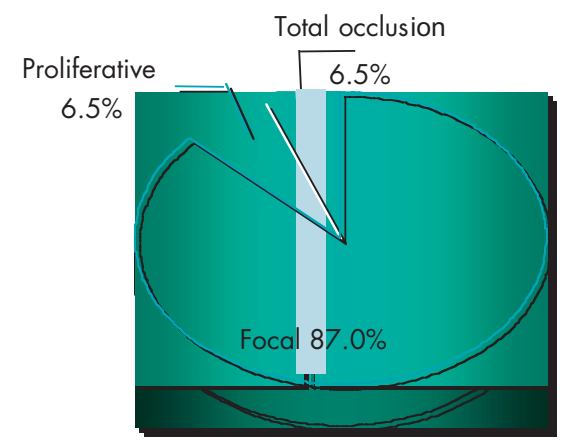

- Margin ISR was noticed in 19 out of 31 ISR pts

- 8 of the 11 "true" ISR were in overlapping stents

- Margin ISR is not seen in a "real world" stenting!

- Diffuse ISR is abolished!

Figure 9 SIRIUS: mode of failure.

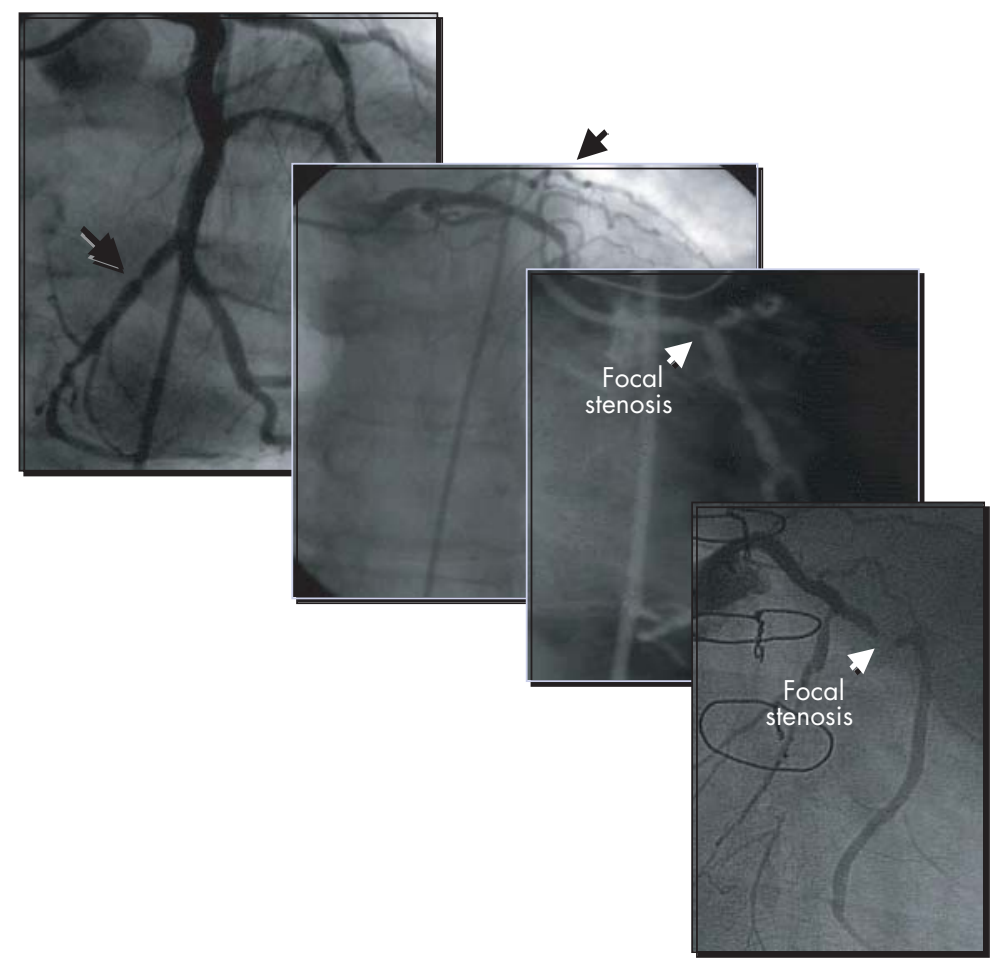


cell surface antigen on EPCs. Early animal data are encouraging with quantitative and semi-quantitative evidence of significantly greater endothelial cells covering those stents coated with the anti-body (fig 8).

Finally absorbable, magnesium alloy stents are being tested by Biotronik. Such stents appear to invoke less of a vessel wall response and less intimal hyperplasia as well as potentially alleviating anxieties about unnecessary longevity of device. Such studies are, however, only in their early stages.

\section{Potential and real problem areas Stent thrombosis}

Concerns regarding excess stent thrombosis in DES (in particular) were raised in 2003/4. Review of the NEW SRIUS data, the e-Cypher registry worldwide implant data $(\mathrm{n}=250$ 000), has shown that the true Cypher stent thrombosis rate is about $0.6 \%$ (no different from bare metal stent) and similar to that seen in the TAXUS IV data. Stent thrombosis therefore appears not to be a problem, although ongoing review with more complex lesions will be mandatory. Even when DES are used in thrombus-rich lesions (acute myocardial infarction, AMI) there appears not to be a problem: in the RESEARCH AMI registry stent thrombosis was not seen in any of the 186 sirolimus patients compared to $1.6 \%$ in those in the bare metal arm. The need for long term antiplatelet treatment and to stop it for non-cardiac procedures is raising some concerns.

\section{Complex lesions}

While we await the final data from the various registries it should be noted that there remain concerns regarding use of DES in left main stem (LMS) lesions. In a small study from Milan the MACE rate was $31 \%$ in unprotected LMS and the question needs to be asked whether ISR may present in the LMS as acute events, unlike elsewhere in the coronary tree. The problems relate in many ways to the technical aspects of safely treating LMS disease. The upcoming SYNTAX trial of TAXUS stents in LMS with or without three vessel disease, comparing outcome to surgical treatment, should help provide an answer, albeit in some years to come. Coronary bifurcation disease remains difficult to treat and is made more so when considering drug eluting stents. " $\mathrm{Y}$ " stenting may leave unprotected balloon injured areas at the origin of the side branch. Rates of restenosis caused by tissue growth in this triangle have reached $30 \%$ in small series. The development of dedicated bifurcation stents, or at least those with side branch access, has not really helped the situation since even-coating with polymer and drug release kinetics have proved difficult to achieve to date. Techniques such as "crush" may result in multiple layers of stent in the main vessel and therefore higher than anticipated drug concentrations at this site. There are still little data on outcomes in vein graft disease and chronic total occlusion. ISR is increasingly being treated with DES although there is little direct evidence to support it. In the DELIVER II registry (paclitaxel with no polymer) there were 527 patients treated for ISR and the TLR rate was $11 \%$. Which is better-ISR treated with vascular brachytherapy or with DES - will hopefully be demonstrated in the TAXUS $\mathrm{V}$ substudy (paclitaxel) and in the SISR (Cypher) trial.

Finally, there is the thorny question of what to do about ISR with DES. Most ISR is likely to be the result of technical problems rather than true DES failures. Data to date suggest that if ISR occurs it is normally focal and may thus be the result of strut failure or inconsistent cover of the stent with drug. For example, in the SIRIUS trial there were 31 ISRs and in 19 the "ISR" was at the margins of the stent (that is, balloon injury with no DES cover). Of the other 11 "true"
ISRs, eight were in overlapping stents. In only two patients from the whole study was the ISR classified as proliferative (fig 9). What to do therefore about ISR with DES becomes clearer. Most would use a balloon (cutting or FX-Minirail to stop balloon movement). Some would place another stent, although there are ongoing studies on paclitaxel delivery balloons (why place another stent?). Vascular brachytherapy will likely become less frequently used and has recently been withdrawn by the company.

\section{DES versus CABG in 2005}

Recently the ARTS 2 trial has been presented. ARTS 1 has compared bare metal stenting with CABG and had shown that CABG was superior almost only because of the need for repeat procedures in the PCI patients. ARTS 2 compared DES use in multivessel disease with the outcomes in ARTS 1. The results clearly showed that use of DES in this non-head to head study was not only better than the bare metal stent arm results in ARTS 1, but also better than the results from the CABG arm. For ARTS 2 versus CABG (ARTS 1) and PCI (ARTS 1), respectively, the results were: death $0.5 \% v 1.8 \% v$ 2.3\%; myocardial infarction $0.7 \% v 3.8 \% v 4.5 \%$; re-PCI $3.1 \% v$ $2.0 \%$ v 7.8\%; any MACE $6.4 \% \vee 9.0 \% \vee 20 \%$ (P Serruys, TCT 2004). The SYNTAX trial is about to start and will be very exciting.

\section{SUMMARY AND CONCLUSIONS}

Drug eluting stents appear to be here to stay and have undoubtedly improved the outcome following stenting. Sirolimus and paclitaxel have lived up to early promise with robust results in increasingly complex trial lesions and in worldwide registries. There is probably little to choose between them, although tissue response suppression favouring the Cypher stent may be balanced by deliverability favouring the TAXUS stent. Other drugs/programme are critical and interestingly while they may have theoretical and even clear practical advantages, are likely to end up being "equivalent" since the size of trial required to demonstrate small clinical benefits would likely require many thousands of patients and be uneconomic to run. Issues such as price and cost effectiveness will be important also and has led to oral rapamycin being reconsidered. Areas requiring scientific resolution include efficacy in complex lesions such as LMS and bifurcation. Finally, developments in stent platform, techniques, and enhanced operator skill need to be recognised as being important. DES will only improve the outcome of the lesions we can (safely) do.

Correspondence to: Dr Anthony H Gershlick, University of Leicester School of Medicine, Glenfield General Hospital, Leicester, UK; agershlick@aol.com

\section{REFERENCES}

1 Serruys PW, Unger F, van Hout BA. The ARTS study (arterial revascularization therapies study). Semin Interv Cardiol 1999;4:209-19.

2 SOS Investigators. Coronary artery bypass surgery versus percutaneous coronary intervention with stent implantation in patients with multivessel coronary artery disease (the stent or surgery trial): a randomised controlled trial. Lancet 2002;360:965-70.

3 Morice MC, Serruys PW, Sousa JE. A randomized comparison of a sirolimuseluting stent with a standard stent for coronary revascularization. N Engl J Med 2002;346:1773-80

4 Moses JW, Leon MB, Popma JJ. Sirolimus-eluting stents versus standard stents in patients with stenosis in a native coronary artery. N Engl J Med 2003;349:1315-23.

5 Schampaert E, Cohen EA, Schluter M. The Canadian study of the sirolimuseluting stent in the treatment of patients with long de novo lesions in small native coronary arteries (C-SIRIUS). J Am Coll Cardiol 2004;43:1110-5.

6 Schofer J, Schluter M, Gershlick AH. Sirolimus-eluting stents for treatment of patients with long atherosclerotic lesions in small coronary arteries: doubleblind, randomised controlled trial (E-SIRIUS). Lancet 2003;362:1093-9.

7 Axel DI, Kunert W, Goggelmann C. Paclitaxel inhibits arterial smooth muscle cell proliferation and migration in vitro and in vivo using local drug delivery. Circulation 1997;96:636-45. 
8 Gershlick A, De Scheerder I, Chevalier B. Inhibition of restenosis with a paclitaxel-eluting, polymer-free coronary stent: the European evaluation of paclitaxel eluting stent (ELUTES) trial. Circulation 2004; 109:487-93

9 Hong MK, Mintz GS, Lee CW. Paclitaxel coating reduces in-stent intimal hyperplasia in human coronary arteries: a serial volumetric intravascular ultrasound analysis from the Asian paclitaxel-eluting stent clinical trial (ASPECT). Circulation 2003;107:517-20.
10 Colombo A, Drzewiecki J, Banning A. Randomized study to assess the effectiveness of slow- and moderate-release polymer-based paclitaxel-eluting stents for coronary artery lesions. Circulation 2003; 108:788-94.

11 Buellesfeld L, Grube E. ABT-578-eluting stents: the promising successor of sirolimus- and paclitaxel-eluting stent concepts? Herz 2004;29:167-70.

12 Formica RN Jr, Lorber KM, Friedman AL. The evolving experience using everolimus in clinical transplantation. Transplant Proc 2004;36(2 suppl):S495-9. 